\title{
Single Tumor Cells With Epithelial-Like Morphology Are Associated With Breast Cancer Metastasis
}

\author{
Liubov A. Tashireva ${ }^{1 *}$, Marina V. Zavyalova ${ }^{1,2}$, Olga E. Savelieva ${ }^{1}$, \\ Tatyana S. Gerashchenko ${ }^{3}$, Evgeniya V. Kaigorodova ${ }^{1,4}$, Evgeny V. Denisov ${ }^{3,5}$ and \\ Vladimir M. Perelmuter ${ }^{1}$

\begin{abstract}
${ }^{1}$ Department of General and Molecular Pathology, Cancer Research Institute, Tomsk National Research Medical Center, Tomsk, Russia, ${ }^{2}$ Department of Pathological Anatomy, Siberian State Medical University, Tomsk, Russia, ${ }^{3}$ Laboratory of Cancer Progression Biology, Cancer Research Institute, Tomsk National Research Medical Center, Tomsk, Russia,

${ }^{4}$ Department of Biochemistry and Molecular Biology, Siberian State Medical University, Tomsk, Russia, ${ }^{5}$ Department of Organic Chemistry, Tomsk State University, Tomsk, Russia
\end{abstract}

Introduction: The identification of tumor cells that can be potential metastatic seeds would reach two key aims - prognosis of metastasis risk and appointment of the optimal adjuvant therapy to prevent metastatic disease. Single tumor cells (STCs) located out of multicellular structures can most likely demonstrate features that are needed to initiate metastasis.

Satyendra Chandra Tripathi,

Edited by: All India Institute of Medical Sciences

Nagpur, India

Reviewed by:

Anna Zaczek,

Medical University of Gdansk, Poland

Jason Somarelli,

Duke University, United States

Saurav Kumar,

Indian Institute of Science (IISC), India

${ }^{*}$ Correspondence:

Liubov A. Tashireva

tashireva@oncology.tomsk.ru

Specialty section:

This article was submitted to Molecular and Cellular Oncology,

a section of the journal

Frontiers in Oncology

Received: 21 August 2019

Accepted: 13 January 2020

Published: 19 February 2020

Citation:

Tashireva LA, Zavyalova MV Savelieva OE, Gerashchenko TS,

Kaigorodova EV, Denisov EV and Perelmuter VM (2020) Single Tumor Cells With Epithelial-Like Morphology

Are Associated With Breast Cancer

Metastasis. Front. Oncol. 10:50.

doi: 10.3389/fonc.2020.00050
Methods: One-hundred-and-thirty-five patients with invasive breast carcinoma of no special type have been enrolled. Molecular subtypes of breast cancer were categorized according to St. Gallen recommendations. Hematoxylin and eosin staining was used to identify STCs with epithelial-like morphology (eSTCs) in breast tumors. Immunofluorescence staining was applied to evaluate stemness and epithelial-mesenchymal transition (EMT) in STCs. The correlation between STCs and recurrence and metastasis-free survival (MFS) was performed using the Kaplan-Meier method and the log-rank test.

Results: Distant metastasis was more frequent in eSTC-positive than eSTC-negative patients (28.0\% vs. 9.4\%, $p=0.007)$. When tumor types were analyzed separately, distant metastasis tended to be more frequent in eSTC-positive than eSTC-negative patients for HER2-positive cancer [75.0\% (3/4) vs. 12.5\% (1/8), $p=0.066]$. In luminal $A[22.7 \%(5 / 22)$ vs. $10.0 \%(3 / 30), p=0.259]$, luminal $B[21.1 \%(4 / 19)$ vs. $6.7 \%(2 / 30), p=0.189]$, and triple-negative $[40.0 \%(2 / 5)$ vs. $11.8 \%(2 / 17)$, $p=0.209]$ cancers, distance metastasis was not associated with eSTCs. Median MFS was not reached in eSTC-positive and eSTC-negative patients. eSTC-positive patients had a higher risk of breast cancer metastasis [hazard ratio (HR) 3.57, 95\% confidence interval $(\mathrm{Cl})$ : 1.46-8.71; $p=0.001]$. When tumor types were analyzed separately, a higher risk of breast cancer metastasis occurred only in HER2-positive patients (HR 8.49, 95\% Cl: 1.29-55.59; $p=0.016)$. Immunofluorescence analysis revealed mesenchymal-like STCs (mSTCs) and inter- and intra-tumor heterogeneity in STCs. There were breast tumors with either ESTCs or mSTCs and tumors with 
both types of STCs. Both eSTCs and mSTCs were represented by cells with different stem and/or EMT phenotypes.

Conclusions: STCs with epithelial-like morphology contribute to breast cancer metastasis and represent an attractive model for studying mechanisms of metastatic seeding. The assessment of STCs in histological sections of breast tumors can be a simple and effective method for the prediction of metastasis risk.

\section{Keywords: single tumor cells, breast cancer, EMT, stem cell, distant metastasis}

\section{INTRODUCTION}

The prediction of tumor progression risk, including lymph node and distant metastasis, remains one of the most important problems in modern oncology. Metastasis can occur not only by single tumor cells (STCs) but also by tumor cell clusters (1). In the past, metastasis was thought to occur by retention of metastatic cells in the capillary system of the first parenchymatous organ encountered (2). This hypothesis was subsequently dismissed as metastatic cells were shown to reach the vasculature of all organs (2). Moreover, clusters of tumor cells are able to pass through capillary-sized vessels (3). Recent findings give further support to the "seed and soil" hypothesis (2) that focuses on the dissemination of STCs.

STCs are a manifestation of intratumor morphological heterogeneity and most likely result from multicellular tumor structures through cancer invasion. In the last years, the appearance of STCs is described as tumor budding in the invasive front (4).

Invasive carcinoma of no special type (IC NST), the most common form of breast cancer (5), is highly heterogeneous in the morphological pattern. Previously, we showed that breast tumor cells can be either single, arranged in small (discrete) groups, or arranged in more complex structures (tubular, alveolar, solid, and trabecular) (6). In addition, we suggested that the intratumor morphological heterogeneity in breast cancer is a result of the unfolding of the invasion program during which epithelial-mesenchymal transition (EMT) leads to significant morphogenetic changes in the tumor landscape: from tubular structures that are close to normal mammary ducts to discrete groups of tumor cells demonstrating a strongly pronounced mesenchymal phenotype (7). The recent study hypothesized that the intratumor morphological heterogeneity can be an attractive model for studying the mechanisms of collective cell invasion (by focusing on solid and trabecular structures) and individual cell invasion (by focusing on discrete groups, namely, STCs) (8).

According to current understanding, STCs may be in a quiescent state or invade by mesenchymal, amoeboid, and hybrid mesenchymal-amoeboid motion $(9,10)$. The definition of these STC states could be an effective tool for studying the mechanisms of cancer invasion and intravasation and would help to predict metastasis risk.

Thus, the aim of this study was to assess morphological and phenotypical heterogeneity of STCs and their prognostic significance in breast cancer patients.
TABLE 1 | Clinicopathological characteristics of breast cancer patients.

\begin{tabular}{|c|c|c|c|c|}
\hline \multicolumn{2}{|c|}{ Characteristics } & \multirow{2}{*}{$\begin{array}{c}\text { No eSTCs, } \\
\text { \% (n) } \\
80(68)\end{array}$} & \multirow{2}{*}{$\begin{array}{c}\text { Yes eSTCs, } \\
\% \text { ( } \boldsymbol{n})\end{array}$} & \multirow{2}{*}{$\begin{array}{l}p \\
\text { NS }\end{array}$} \\
\hline $\begin{array}{l}\text { Postoperative } \\
\text { treatment }\end{array}$ & $\begin{array}{l}\text { Adjuvant chemotherapy: } \\
\text { CMF, FAC, CAX }\end{array}$ & & & \\
\hline & $\begin{array}{l}\text { Antiestrogen therapy } \\
\text { with tamoxifen }\end{array}$ & $20(17)$ & $18(9)$ & NS \\
\hline \multirow[t]{3}{*}{ Age } & $<35$ years & $35(30)$ & $26(13)$ & NS \\
\hline & $35-50$ years & $22(19)$ & $36(18)$ & NS \\
\hline & $>50$ years & $43(36)$ & $38(19)$ & NS \\
\hline \multirow{2}{*}{$\begin{array}{l}\text { Menopausal } \\
\text { status }\end{array}$} & Premenopausal & $33(28)$ & $36(18)$ & NS \\
\hline & Postmenopausal & $67(57)$ & 64 (32) & NS \\
\hline \multirow[t]{6}{*}{ Stage } & I $\left(T_{1} N_{0} M_{0}\right)$ & $28(24)$ & $16(8)$ & NS \\
\hline & $\| A\left(T_{0-1} N_{1} M_{0}, T_{2} N_{0} M_{0}\right)$ & $39(33)$ & $32(16)$ & NS \\
\hline & $\| B\left(T_{2} N_{1} M_{0}, T_{3} N_{0} M_{0}\right)$ & $13(11)$ & $14(7)$ & NS \\
\hline & $\begin{array}{l}\text { IIIA }\left(T_{0-2} N_{2} M_{0}\right. \\
\left.T_{3} N_{1-2} M_{0}\right)\end{array}$ & $14(12)$ & $30(15)$ & NS \\
\hline & IIIB $\left(\mathrm{T}_{4} \mathrm{~N}_{0-2} \mathrm{M}_{0}\right)$ & $1(1)$ & $0(0)$ & NS \\
\hline & IIIC $\left(T_{1-4} \mathrm{~N}_{3} \mathrm{M}_{0}\right)$ & $5(4)$ & $8(4)$ & NS \\
\hline \multirow[t]{3}{*}{ Grade } & I & $7(6)$ & $10(5)$ & NS \\
\hline & $\|$ & $74(63)$ & $80(40)$ & NS \\
\hline & III & $19(16)$ & $10(5)$ & NS \\
\hline \multirow[t]{3}{*}{ Tumor size } & $<2 \mathrm{~cm}$ & $55(47)$ & $30(15)$ & 0.007 \\
\hline & $2-5 \mathrm{~cm}$ & $44(37)$ & $62(31)$ & 0.049 \\
\hline & $>5 \mathrm{~cm}$ & $1(1)$ & $8(4)$ & NS \\
\hline \multirow{4}{*}{$\begin{array}{l}\text { Molecular } \\
\text { subtype }\end{array}$} & Luminal A & $35(30)$ & $44(22)$ & NS \\
\hline & Luminal B & $35(30)$ & $38(19)$ & NS \\
\hline & Triple-negative & $20(17)$ & $10(5)$ & NS \\
\hline & HER2-positive & $10(8)$ & $8(4)$ & NS \\
\hline \multirow{2}{*}{$\begin{array}{l}\text { Estrogen } \\
\text { receptors }\end{array}$} & Positive & $75(64)$ & $86(43)$ & NS \\
\hline & Negative & $25(20)$ & $14(7)$ & NS \\
\hline \multirow{2}{*}{$\begin{array}{l}\text { Progesterone } \\
\text { receptors }\end{array}$} & Positive & $62(53)$ & $64(32)$ & NS \\
\hline & Negative & $38(32)$ & $36(18)$ & NS \\
\hline \multirow[t]{2}{*}{ HER2 } & Positive & $20(17)$ & $26(13)$ & NS \\
\hline & Negative & $80(68)$ & $74(37)$ & NS \\
\hline \multirow[t]{2}{*}{ Ki-67 } & Expression < 20\% & $36(31)$ & $48(24)$ & NS \\
\hline & Expression > 20\% & $64(54)$ & $52(26)$ & NS \\
\hline
\end{tabular}

CMF, cyclophosphamide, methotrexate, 5-fluorouracil; FAC, 5-fluorouracil, adriamycin, cyclophosphamide; CAX, cyclophosphamide, adriamycin, xeloda; NS, not significant. 


\section{MATERIALS AND METHODS}

\section{Patients and Specimens}

The retrospective study included 135 patients with IC NST (stage I-IIIC, $\mathrm{T}_{1-4} \mathrm{~N}_{0-3} \mathrm{M}_{0}$ ) who were treated in the Cancer Research Institute, Tomsk NRMC between 2008 and 2015 (Table 1). The median age was 55 years (range: $29-85$ years). All cases were reexamined, and IC NST was diagnosed and staged according to the World Health Organization's recommendations (5). Patients had not received neoadjuvant chemotherapy and were monitored using computed tomography (CT) scan every 6 months to identify metastatic lesions. Recurrence- and metastasis-free survival (RFS and MFS) was defined as the time window spanning between the diagnosis and the detection of the first recurrence or metastatic lesion on imaging or patient death, whichever occurred first.

Formalin-fixed, paraffin-embedded (FFPE) samples of breast tumors were used for morphological $(n=135)$, immunohistochemical $(n=135)$, and immunofluorescence analyses $(n=25)$.

The procedures followed in this study were in accordance with the Helsinki Declaration (1964, amended in 1975 and 1983). All patients signed informed consent for voluntary participation. The study was approved by the review board of the Cancer Research Institute, Tomsk NRMC on 17 June 2016 (the approval number is 8 ).

\section{Morphological Analysis}

The morphological analysis included the determination of STCs in breast tumors (Figure 1). Five-micrometer-thick hematoxylin and eosin (H\&E)-stained sections of FFPE samples were used for the STC analysis using an Axio Lab.A1 light microscope (Carl Zeiss, Germany). STCs (or detached individual tumor cells) were determined in the entire tumor tissue in contrast to tumor buds residing in the invasive front and defined as tumor cells located out of multicellular tumor structures (tubular, alveolar, solid, and trabecular) but similar to them in cytological features. STCs with epithelial morphology (eSTCs) had eosinstained cytoplasm of different volumes and were larger than immune and stromal cells (tumor cell nuclei $\geq 3 \times$ the size of lymphocyte). Tumor cells similar to fibroblasts/myofibroblasts or mononuclear leukocytes (lymphoid cells, macrophages) in shape and size were not possible to identify in H\&E-stained sections and were revealed using the epithelial marker, cytokeratin 7 (CK7). We attributed these cells to STCs with mesenchymal morphology (mSTCs).

\section{Immunohistochemical Analysis}

Immunohistochemistry was used to assess the expression of estrogen and progesterone receptors (ER and PR),

TABLE 2 | Frequency of recurrences in breast cancer patients with eSTCs.

\begin{tabular}{lccc}
\hline & No eSTCs & Yes esTCs & $\boldsymbol{P}$-values \\
\hline Luminal A & $3.33(1 / 30)$ & $4.54(1 / 22)$ & 1.000 \\
Luminal B & $0.00(0 / 30)$ & $0.00(0 / 19)$ & 1.000 \\
Triple-negative & $5.88(1 / 17)$ & $4.00(2 / 5)$ & 0.116 \\
HER2-positive & $12.50(1 / 8)$ & $0.00(0 / 4)$ & 1.000 \\
\hline
\end{tabular}

P-values indicate differences between patients with the absence ("No") and presence ("Yes") of eSTCS.
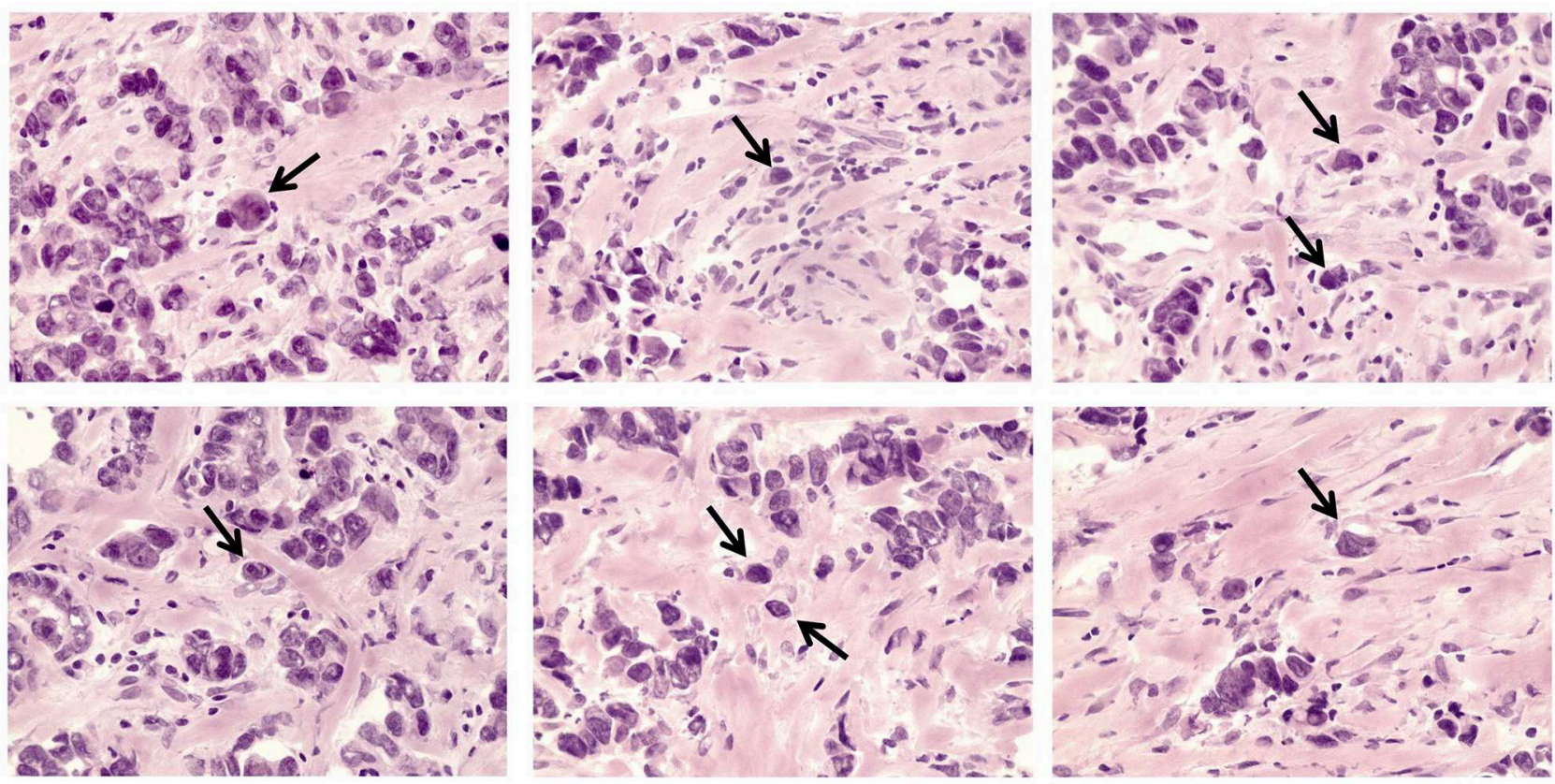

FIGURE 1 | eSTCs in invasive breast carcinoma of no special type. Arrows indicate eSTCs located out of multicellular structures. $400 \times$ magnification. 
HER2, and Ki-67 in breast tumors using the following antibodies: mouse anti-ER (Dako, Cat. \# IR084, clone 1D5, RTU), mouse anti-PR (Dako, Cat. \# IR068, clone PgR636, RTU), rabbit anti-HER2 (Dako, Cat. \# A0485, 1:800), and mouse anti-Ki-67 (Dako, Cat. \# IR626, clone MIB-1, RTU). Immunohistochemistry was performed as previously described (11). ER and PR immunostaining was scored using ASCO/CAP Recommendations (12). HER2 immunostaining was scored using St. Gallen recommendations (13). Ki-67 immunostaining was expressed as the percentage of positively stained cells. At least 10 fields of view and at least 1,000 cells at $400 \times$ magnification (field area $=0.196 \mathrm{~mm}^{2}$ ) were analyzed per sample. Molecular subtypes of the IC NST were categorized according to St. Gallen recommendations (13): luminal $\mathrm{A}_{\left(\mathrm{ER}^{+}\right.}$and/or $\mathrm{PR}^{+}, \mathrm{HER} 2^{-}$, and $\mathrm{Ki}-67<20 \%)$, luminal $\mathrm{B}\left(\mathrm{ER}^{+}\right.$and/or $\mathrm{PR}^{+}, \mathrm{HER} 2^{-/+}$, and $\mathrm{Ki}-67 \geq 20 \%$ ), HER2-positive $\left(\mathrm{ER}^{-}, \mathrm{PR}^{-}\right.$, and HER2 $\left.{ }^{+}\right)$, and triple-negative $\left(\mathrm{ER}^{-}, \mathrm{PR}^{-}\right.$, and $\left.\mathrm{HER} 2^{-}\right)$.

\section{Immunofluorescence Analysis}

Immunofluorescence staining was used to analyze the morphological and phenotypical heterogeneity of STCs. Seven-micrometer-thick sections were prepared from FFPE tumor samples $(n=25)$, deparaffinized, rehydrated, processed for heat-induced epitope retrieval in PT Link (Dako, Denmark) with high $\mathrm{pH}$ buffer, and blocked with $3 \%$ bovine serum albumin (Amresco, USA) in PBS. Subsequently, the sections were incubated with a cocktail of primary antibodies: mouse anti-CD133 (MyBioSource, Cat. \# MBS5305439, clone 3F10, 1:800), rabbit anti-Snail/Slug (Abcam ab180714, 1:400), and goat anti-CK7 (Santa Cruze, Cat. \# sc-70936, 1:50) or mouse anti-CD133 (MyBioSource, Cat. \# MBS5305439, 1:800), rabbit anti-N-cadherin (Abcam ab76057, 1:400), and goat anti-CK7 (Santa Cruze, Cat. \# sc-70936, 1:50) followed by incubation with the appropriate secondary antibodies: goat anti-mouse IgG H\&L (Alexa Fluor 488, Abcam ab150117, 1:200), goat
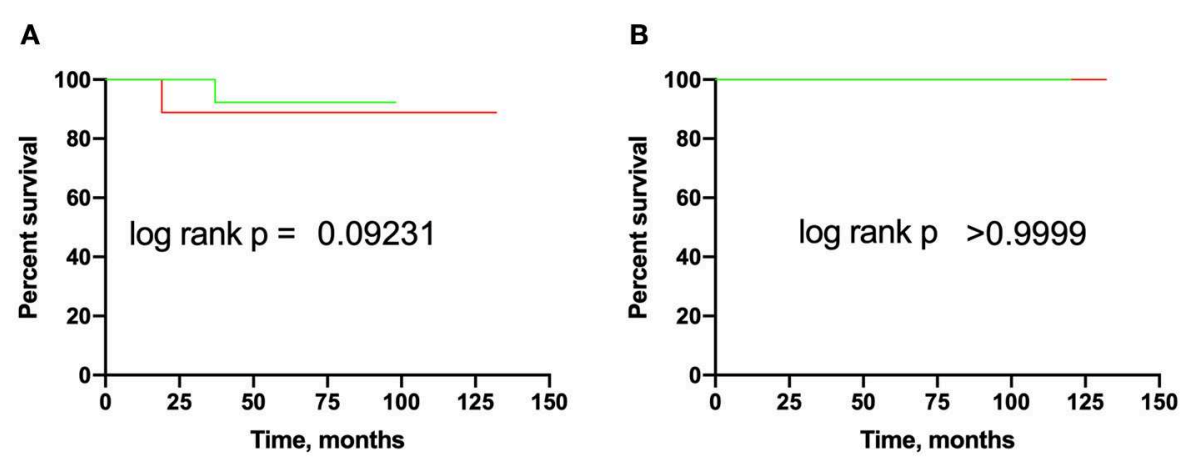

C

D
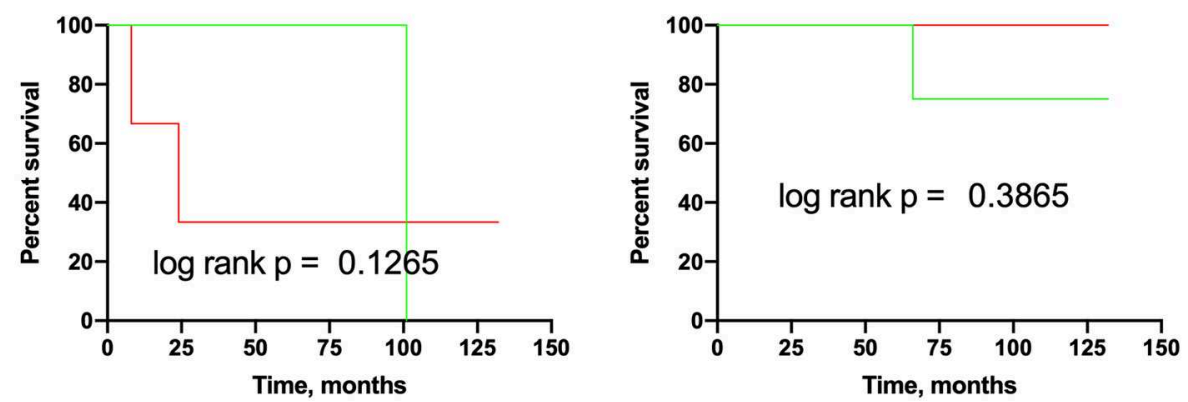

E

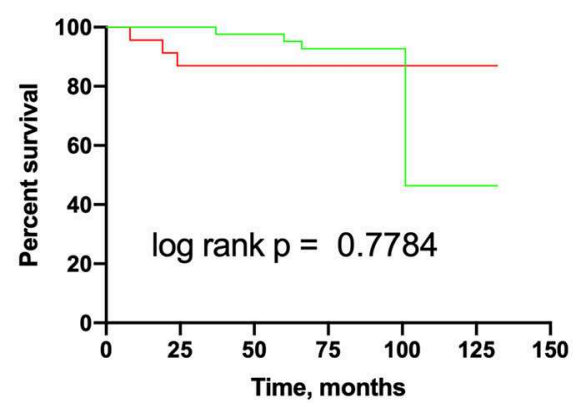

No STCs

— Yes STCs

FIGURE 2 | Recurrence-free survival in breast cancer patients with eSTCs. (A) Luminal A. (B) Luminal B. (C) Triple-negative. (D) HER2-positive. (E) Total group. 
anti-rabbit IgG H\&L (Cy3, Abcam ab6939, 1:200), and donkey anti-goat IgG H\&L (Alexa Fluor 647, Abcam ab150135, 1:200). Finally, Vectashield mounting medium (Vector Laboratories, USA) containing DAPI was used to detect nuclei and mount the specimens. The samples were analyzed using an LSM 780 NLO confocal microscope (Carl Zeiss, Germany).

Normal endometrial, liver, and tonsillar tissues were used as a positive control for anti-CD133, N-cadherin, and Snail antibodies, respectively. Human skin fibroblasts were used as a negative control for CD133 and N-cadherin staining. Snail expression was heterogeneous in cells of mammary acini and ducts. Negative control for Snail staining was acinar and ductal cells that did not express this protein.

Using CK7 staining, we identified STCs with distinct epithelial- and mesenchymal-like morphologies. eSTCs had abundant cytoplasm and were larger than immune and stromal cells (tumor cell nuclei $\geq 3 \times$ the size of the lymphocyte). mSTCs were detected as CK7-positive cells, in which the size and the cytological characteristics were similar to those of immune and stromal cells.
CD133-positive STCs were designated as stem cells. Snail was considered as a marker of early EMT (14), whereas N-cadherinas a marker of advanced EMT $(15,16)$. Two parameters were used to evaluate the distribution of cells with stem and EMT phenotypes in eSTCs and mSTCs. First, we assessed how often stem and EMT cells were observed in eSTC- and mSTC-positive patients. Second, we calculated the percentage of stem and EMT cells among eSTCs and mSTCs.

\section{Statistical Analysis}

Statistical analysis was performed using STATISTICA 8.0 for Windows (StatSoft Inc., USA). Normal distribution was tested using the Shapiro-Wilk test. Fisher's exact test was applied to assess differences in the frequency of cell subpopulations both between STCs with various morphologies and different clinicopathological parameters. The Mann-Whitney U-test was applied to analyze differences in the percentage of cell subpopulations between STCs with various morphologies. MFS and RFS were evaluated using the Kaplan-Meier estimator with the log-rank test. Differences were considered significant at
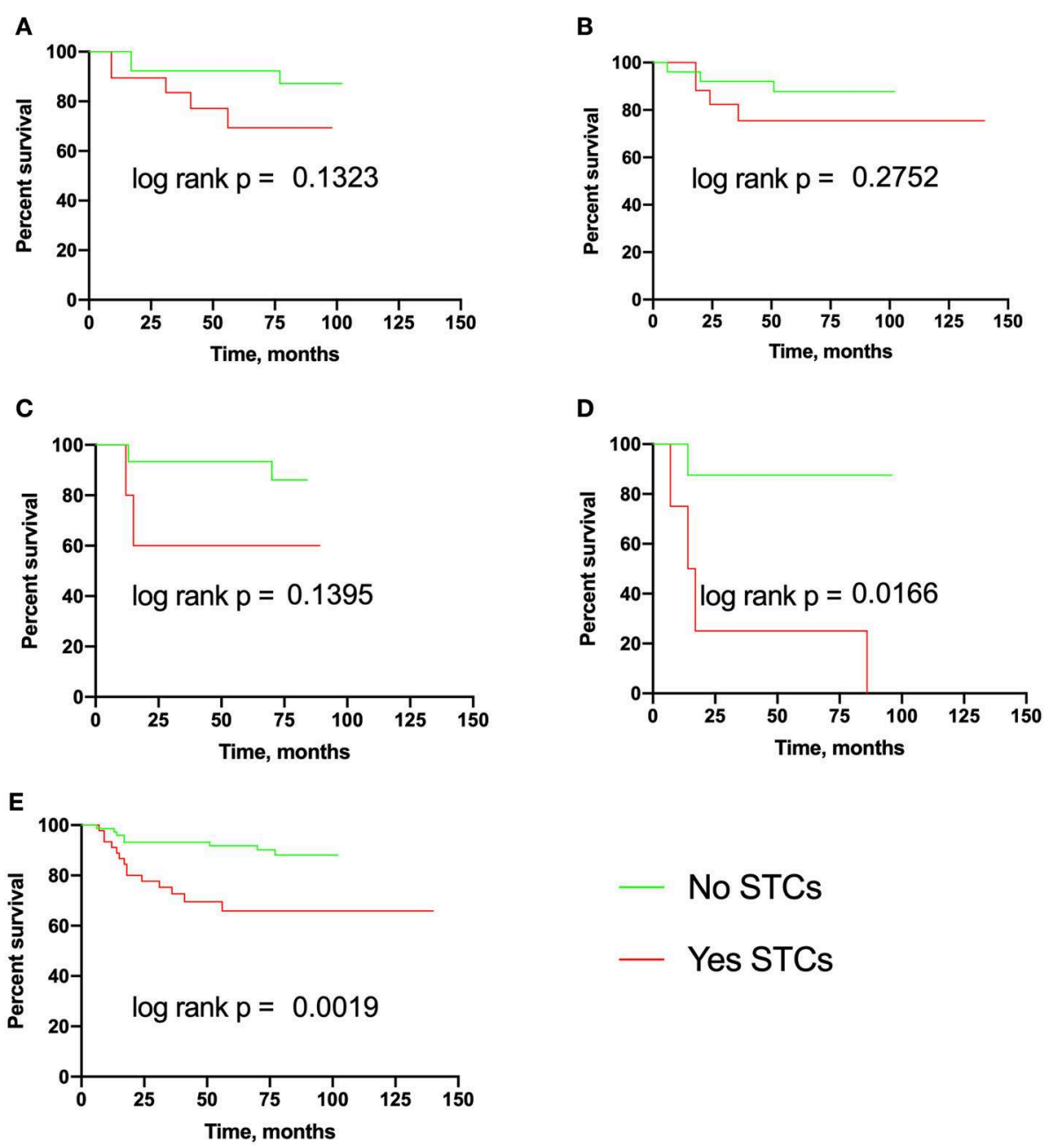

FIGURE 3 | Metastasis-free survival in breast cancer patients with eSTCs. (A) Luminal A. (B) Luminal B. (C) Triple-negative. (D) HER2-positive. (E) Total group. 

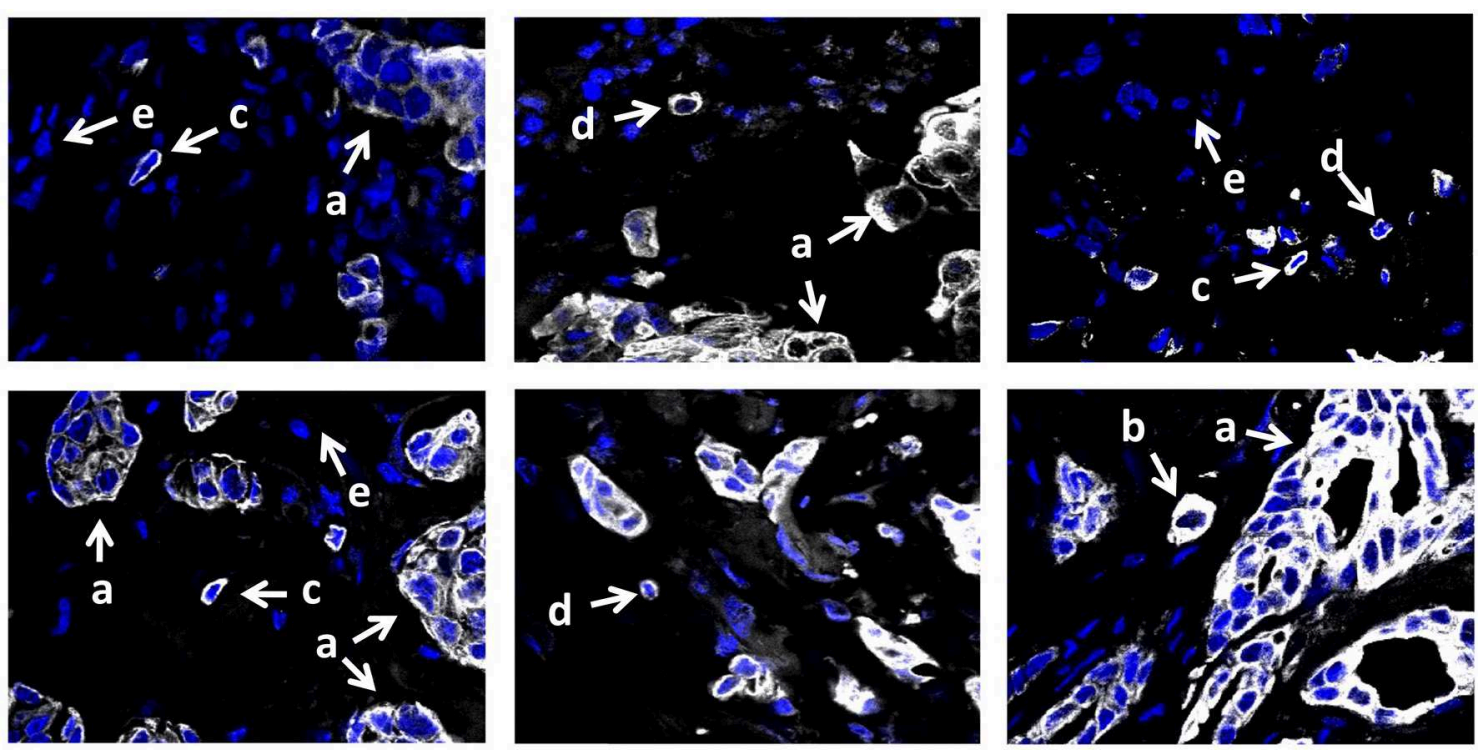

FIGURE 4 | CK7 expression in invasive breast carcinoma of no special type. Multicellular structures are formed by tumor cells with epithelial morphology (marked by the letter "a"). Few CK7-positive STCs have epithelial morphology and do not differ from tumor cells of multicellular structures (marked by the letter "b"). Numerous CK7-positive mSTCs with fibroblast- (marked by the letter "c") or lymphocyte-like (marked by the letter "d") morphology are similar to CK7-negative stromal cells (marked by the letter "e"). $400 \times$ magnification.

$p<0.05$. Differences at $0.05>p<0.1$ were discussed as non-significant trends. Cox proportional hazard analysis was used to assess the association between eSTCs and MFS and RFS. Associations were reported as hazard ratios (HRs) with 95\% confidence intervals (95\% CIs) and p-values (likelihood ratio test).

\section{RESULTS AND DISCUSSION}

\section{Frequency of eSTCs in Breast Cancer: an Association With Clinicopathological Parameters}

Here, we assessed the frequency of STCs in breast tumors ( $n$ $=135$ ). It must be noted that only eSTCs could be detected in the $\mathrm{H} \& \mathrm{E}$ sections of breast tumors (Figure 1). eSTCs were found in $37.0 \%(50 / 135)$ of the breast tumors. Their frequency did not vary between molecular subtypes of breast cancer: $42.3 \%$ (22/52)-luminal A, 38.8\% (19/49)-luminal B, 22.7\% (5/22)triple-negative, and $33.3 \%$ (4/12)-HER2-positive tumors. The frequency of eSTCs did not depend on the parameters of the patients. However, eSTCs were more frequent in large-sized tumors $(2-5 \mathrm{~cm})$ (Table $\mathbf{1})$.

\section{Association of eSTCs With Breast Cancer Progression}

In this section, we assessed the association of eSTCs with recurrence, lymph node, and distant metastasis in breast cancer. It turned out that the probability of recurrence and RFS did not depend on eSTCs (Table 2, Figure 2).
By contrast, lymph node involvement was more frequent in eSTC-positive than in eSTC-negative patients $[60.0 \%(30 / 50)$ vs. $32.1 \%(27 / 84), p=0.002]$. This association was significant only in luminal A [59.1\% (13/22) vs. $23.3 \%(7 / 30), p=0.011]$. In luminal B [63.1\% (12/19) vs. $44.8 \%(13 / 29), p=0.250]$, triple-negative [60.0\% (3/5) vs. $29.4 \%(5 / 17), p=0.308]$, and HER2-positive cancer $[50.0 \%(2 / 4)$ vs. $25.0 \%(2 / 8), p=0.547]$, lymph node metastasis was not associated with eSTCs.

The frequency of distant metastasis was also higher in eSTCpositive than in eSTC-negative patients [28.0\% (14/50) vs. 9.4\% (8/85), $p=0.007]$. This association was at a borderline significance in HER2-positive [75.0\% (3/4) vs. $12.5 \%(1 / 8), p=$ $0.066]$ cancer and not significant in luminal A $[22.7 \%(5 / 22)$ vs. $10.0 \%(3 / 30), p=0.259]$, luminal B [21.1\% (4/19) vs. $6.7 \%(2 / 30)$, $p=0.189]$, and triple-negative [40.0\% (2/5) vs. $11.8 \%(2 / 17), p=$ 0.209] cancers.

The median MFS was not reached in eSTC-positive and eSTCnegative patients both in the total group and in any molecular subtypes (Figure 3). eSTC-positive patients had a higher risk of breast cancer metastasis (HR 3.57, 95\% CI: 1.46-8.71; $p=0.001$ ). When tumor types were analyzed separately, a higher risk of breast cancer metastasis occurred only in HER2-positive patients (HR 8.49, 95\% CI: 1.29-55.59; $p=0.016$ ).

\section{Morphological Heterogeneity of STCs}

To assess the morphological heterogeneity in STCs, we analyzed the expression of the epithelial marker, cytokeratin 7 (CK7), in 15 breast cancers with eSTCs and 10 cases without these cells. CK7-positive STCs were represented by cells with both distinct epithelial and mesenchymal (fibroblast- or lymphocytelike) morphologies (Figure 4). In particular, $40.0 \%$ (10/25) of the 
cases had eSTCs, $28.0 \%$ (7/25) of the cases had mSTCs, and 32.0\% $(8 / 25)$ of the cases had eSTCs and mSTCs simultaneously.

CK7-positive STCs tended to be observed more frequently in luminal B cancers, whereas CK7-negative STCs were more often in triple-negative cancers (Table 3). In HER2-positive cancer, the frequencies of $\mathrm{CK}^{+}$and $\mathrm{CK}^{-}$STCs were not compared due to small patient numbers in each group (Table 3 ).

Morphological and immunofluorescence analysis showed a high level of concordance (88\%) in the identification of eSTCs. In $12 \%(3 / 25)$ of the cases, eSTCs were not detected morphologically but were observed by immunofluorescence staining with CK7. Most likely, it was related to the scarcity of CK7-positive STCs in $\mathrm{H} \& \mathrm{E}$ sections or their intermediate epithelial-mesenchymal morphology. It must be noted that immunofluorescence analysis not only confirmed the absence of eSTCs in H\&E stained sections of some cases but also showed the presence of mSTCs in these cases.

Based on the morphological analysis, we classified breast cancer patients to three groups: with eSTCs (mSTCs-) only, with mSTCs (eSTCs-) only, and with eSTCs and mSTCs $(\mathrm{eSTC}+\mathrm{mSTC}+)$ simultaneously.

TABLE 3 | Frequency of eSTCs and mSTCs in different molecular subtypes of breast cancer.

\begin{tabular}{lcccc}
\hline Patient groups & & eSTC+mSTC- & eSTC-mSTC+ & eSTC+mSTC+ \\
\hline Luminal A & 1 & $20.0(2 / 10)$ & $30.0(3 / 10)$ & $50.0(5 / 10)$ \\
Luminal B & 2 & $\begin{array}{c}66.7(6 / 9) \\
\mathrm{p}_{2-1}=0.069\end{array}$ & $11.1(1 / 9)$ & $22.2(2 / 9)$ \\
& & $\mathrm{p}_{2-3}=0.181$ & & \\
Triple-negative & 3 & $0.0(0 / 3)$ & $66.7(2 / 3)$ & $33.3(1 / 3)$ \\
& & & $\mathrm{p}_{3-2}=0.127$ & \\
\hline
\end{tabular}

Fisher's exact test. $p_{2-1}, p_{2-3}$, and $p_{3-2}$, differences between luminal $B(2)$, luminal $A$ (1), and triple-negative (3) cancers.

\section{Heterogeneity of STCs in Stem and EMT Features}

Markers for stemness (CD133) and EMT (Snail and/or Ncadherin) were assessed in eSTCs and mSTCs (Table 4, Figures 5, 6). However, the frequencies of tumors with stem and/or EMT cells did not differ between patient groups, eSTC (mSTC-) and mSTC (eSTC-) (Table 4). Nevertheless, the differences in the percentages of different subpopulations were observed among eSTCs and mSTCs (Table 5).

Stem cells with early $\left(\mathrm{CK}^{+}{ }^{+} \mathrm{CD} 133^{+}{ }^{\text {Snail }}{ }^{+}\right)$and late $\left(\mathrm{CK} 7{ }^{+} \mathrm{CD} 133^{+} \mathrm{N}\right.$-cadherin $\left.{ }^{+}\right)$EMT were lower in the mSTC (eSTC-) group compared to the eSTC (mSTC-) group (Table 5). In the patient group with the simultaneous presence of eSTCs and mSTCs, $\mathrm{CK}^{+} \mathrm{CD}_{13}{ }^{+}{ }^{\text {Snail }}{ }^{-}$and $\mathrm{CK}^{+} \mathrm{CD} 133^{+} \mathrm{N}-$ cadherin $^{-}$cells tended to be rare among mSTCs compared to eSTCs (Table 4). Surprisingly, the percentage of these stem-like non-EMT cells did not differ between eSTCs and mSTCs in patients in which the tumor simultaneously contained eSTCs and mSTCs (Table 5).

Non-stem and non-EMT $\left(\mathrm{CK}^{+}{ }^{+} \mathrm{CD} 133^{-}\right.$Snail $\left.{ }^{-}\right)$cells were predominant among eSTCs and mSTCs in all three groups of patients: eSTC (mSTC-), mSTC (eSTC-), and eSTC+mSTC+ (Tables 4, 5).

\section{DISCUSSION}

STCs, i.e., detached individual tumor cells, are widely recognized by pathologists in H\&E-stained sections, but their phenotypic features and role in cancer progression remain to be elucidated. Some studies reported genetic analysis of STCs, but in many cases, these were not detached individual cells and were obtained from tumor samples by mechanical dissociation (17) or from multicellular structures by laser microdissection (18). Other studies described STCs at the invasive front, for example, in

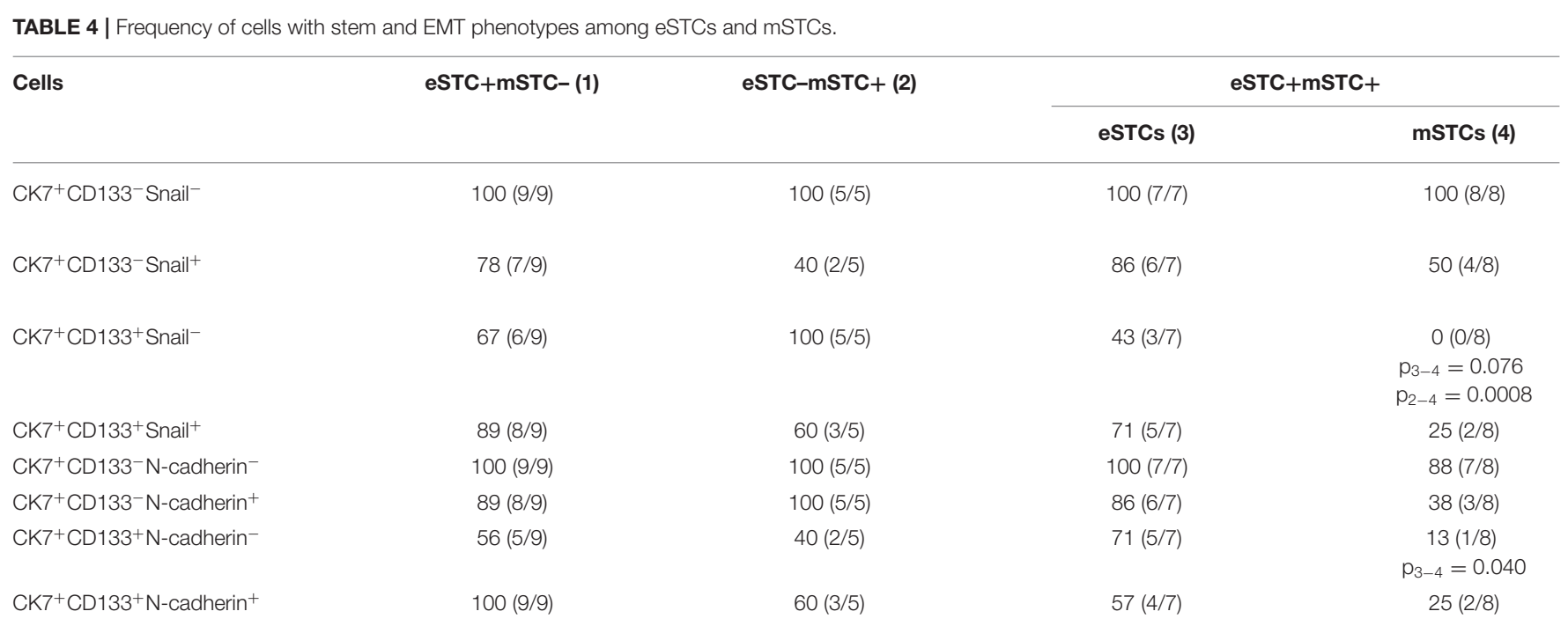

\footnotetext{
Fisher's exact test. $p_{2-4}$ and $p_{3-4}$, differences between groups of patients with only mSTCs (2) and simultaneously with eSTCs (3) and mSTCs (4).
} 


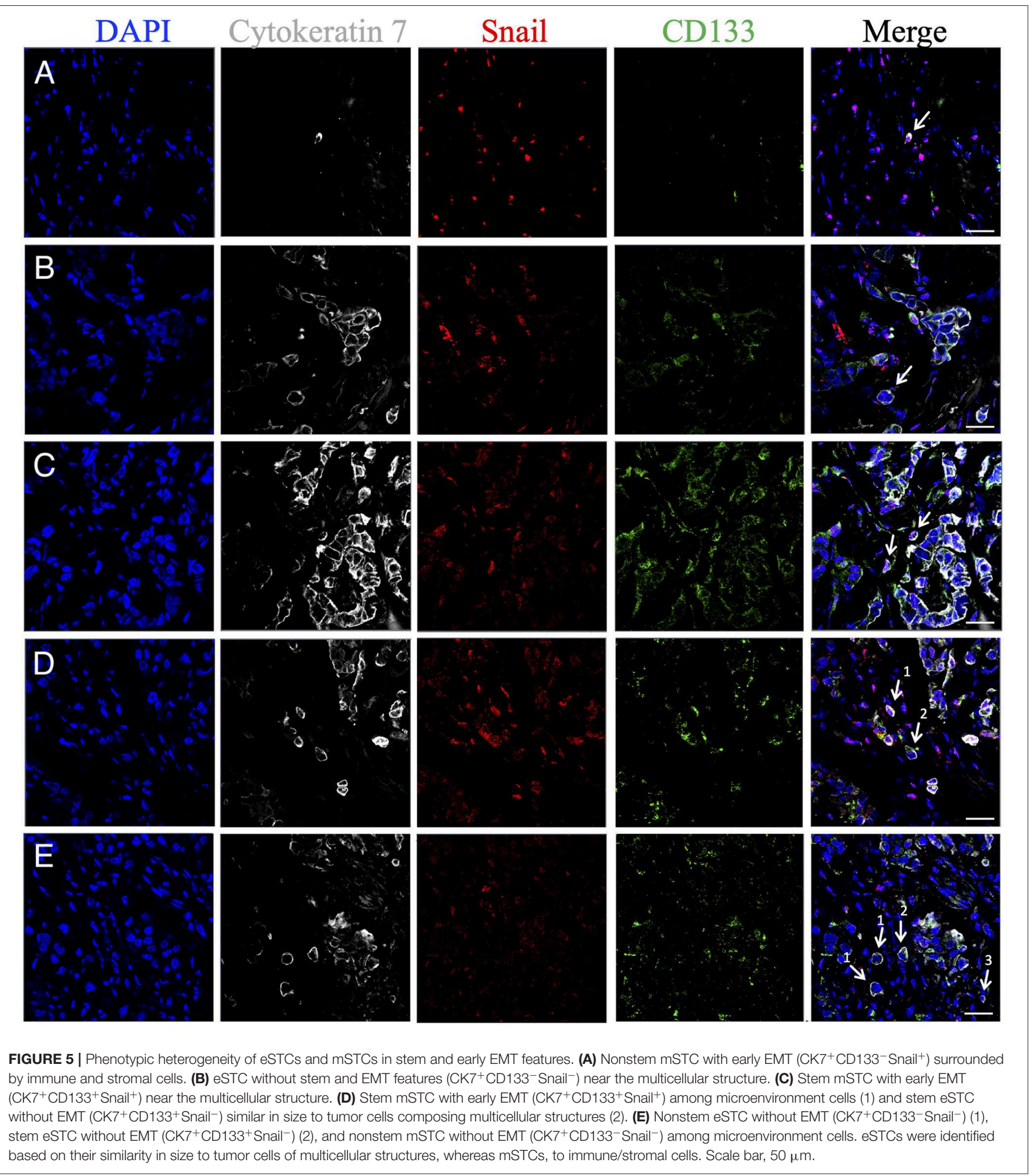

tumor budding (4), and investigated their genomic copy number profiles (19).

In this study, we determined STCs in the entire tumor tissue as cells with epithelial- or mesenchymal-like morphology that were represented by subpopulations with various EMT and stem phenotypes. However, only eSTCs were associated with breast cancer metastasis. In addition, eSTCs were prevalent in largesized breast tumors. This finding may explain why large breast 


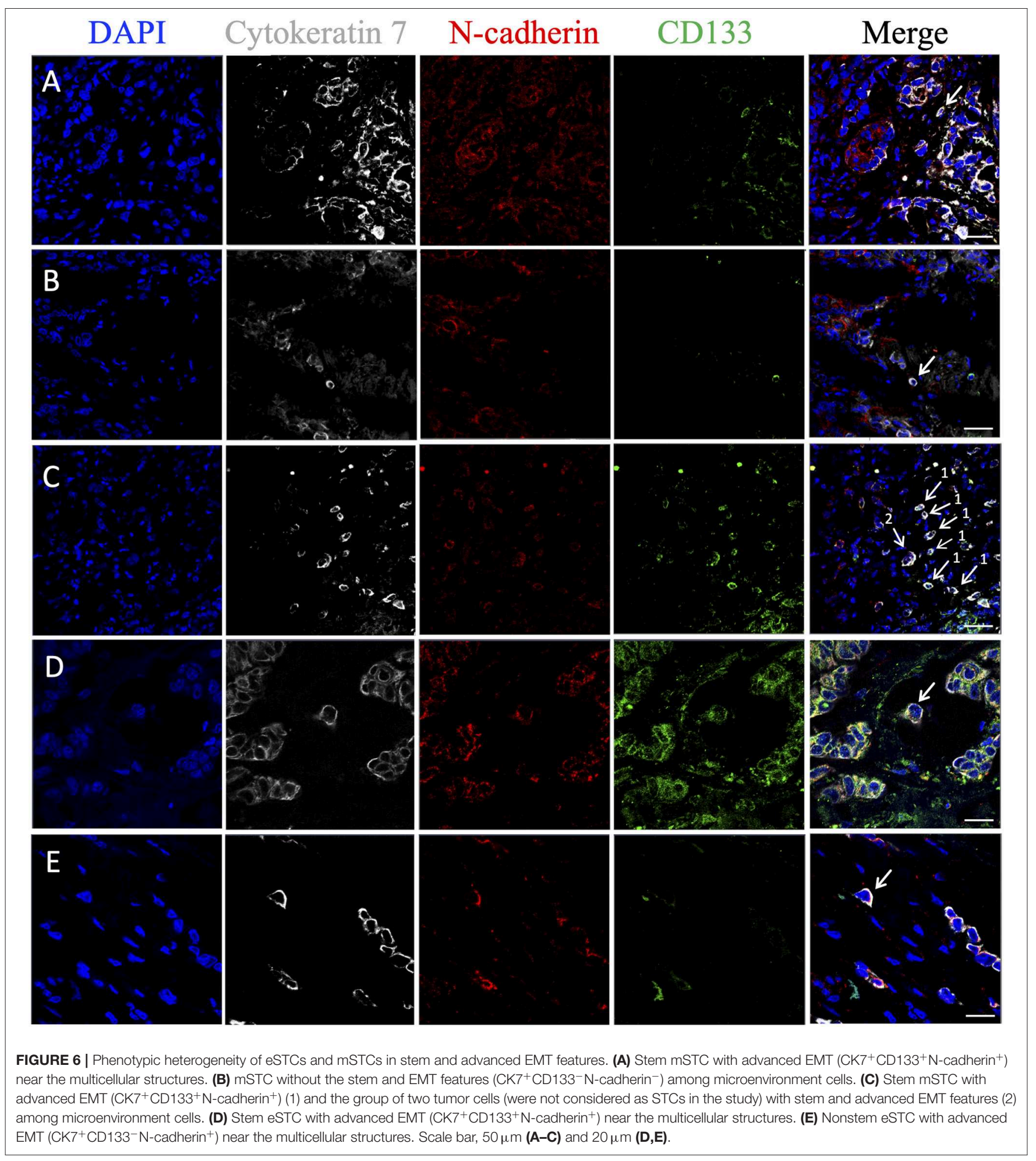

tumors metastasize more often than small tumors (20). STCs probably appear in the tumor by detaching from multicellular tumor structures. Most likely, it may occur through two different mechanisms. The first mechanism, demonstrated by various studies, is that STCs are a result of detaching of leader cells at the invasive edge of multicellular tumor structures (21). The second mechanism is rather hypothetical and may be due to the pushing of tumor cells from multicellular structures to the stroma. As suggested by Rosenblatt and coauthors, such basal extrusion occurs in conditions of disrupting the S1P-S1P2 signaling 
TABLE 5 | Percentage of cells with stem and EMT phenotypes among eSTCs and mSTCs.

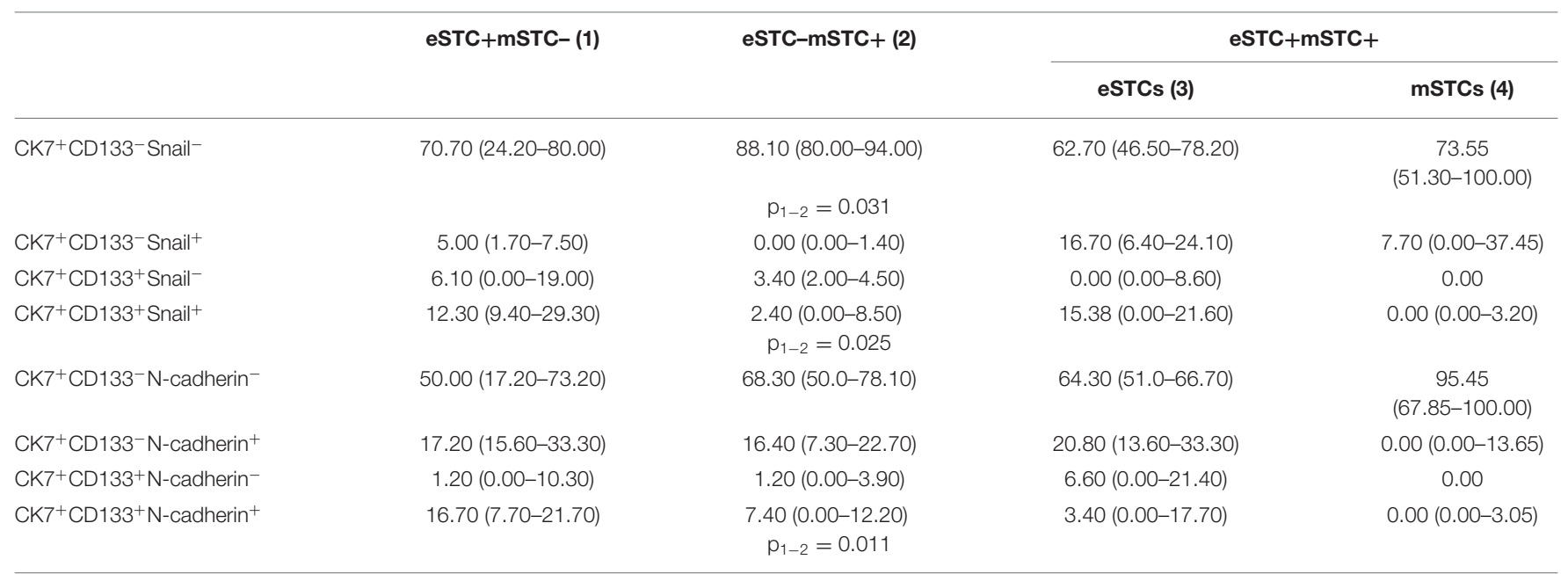

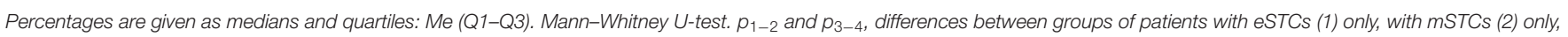
and with eSTCs (3) and mSTCs (4) simultaneously.

pathway underlying physiologically normal apical extrusion of cells that completed their life cycle $(22,23)$.

It is reasonable to assume that detaching cells from multicellular structures, or collective-individual transition, should be accompanied by EMT and following the inhibition of cell-cell adhesion. However, our results show that most STCs are not characterized by the expression of Snail or N-cadherin. This can be explained either by the fact that basal extrusion, if it occurs, is not related to EMT or by EMT reversibility (i.e., mesenchymal-epithelial transition, MET). In fact, the study of EMT in cancers resulted in the understanding of this process as a consecutive spectrum of cell states from initial epithelial through intermediate hybrid or metastable to terminal mesenchymal phenotypes $(24,25)$.

The term "epithelial-mesenchymal plasticity," which is more and more often used at the present time, most accurately describes the EMT-MET interconversion, with the possibility of phenotypic changes from epithelial to mesenchymal states and vice versa with a stop at any stage of the process $(25,26)$. The presence of cells with varying degrees of EMT among STCs with epithelial and mesenchymal morphology most likely reflects epithelial-mesenchymal plasticity.

At present, it is known that complete EMT is less effective for cancer progression than partial EMT that retains the molecular and morphological features of epithelial cells (26). Tumor cells with a hybrid epithelial-mesenchymal phenotype are more adaptive to the tumor microenvironment and resistant to immune reactions and demonstrate a pronounced colonyforming ability (27). In our study, eSTCs expressing Snail or Ncadherin most likely possess a hybrid (metastable) phenotype. This can explain the significant association between eSTCs and high probability of breast cancer metastasis. In addition, our results indicate that the presence of EMT features, particularly Snail or N-cadherin expression, is not accompanied by an obligatory transition from epithelial to fibroblast- or lymphocyte-like cell shape. The absence of spindle-like shape in tumor cells undergoing EMT was reported previously (28).

Nevertheless, tumor cells with mesenchymal morphology can be identified in $\mathrm{H} \& \mathrm{E}$ sections if they are located in multicellular structures together with epithelial-like cells. For example, the three-dimensional reconstruction of tumor tissue sections showed that tumor cells located at the invasive front of the collective invading structure rarely have spindle-like or round (mesenchymal) shape (29). In histological specimens, it is almost impossible to observe STCs with fibroblast- or lymphocyte-like morphology without epithelial markers. This fact should be considered when a pathologic response to neoadjuvant chemotherapy is assessed. It is well-known that a pathologic complete response ( $\mathrm{pCR}$ ) is a favorable prognostic factor. However, because pCR is determined by a pathologist based on the assessment of H\&E sections, STCs with fibroblastor lymphocyte-like morphology cannot be detected and the diagnosis can be inaccurate. Despite a high probability of this mistake, pCR remains a marker of good prognosis. Does it mean that mSTCs are not significant for cancer progression? The association between eSTCs and high frequency of breast cancer metastasis probably confirms the low importance of mSTCs in the formation of metastases. In reality, tumor cells with fibroblast-like shape were found to have a decreased aggressiveness (30). However, future studies should clarify the significance of eSTCs and mSTCs in metastasis.

According to our study, some eSTCs and mSTCs demonstrated features of either stemness, EMT, or simultaneous stemness and EMT that make them similar to circulating tumor cells (CTCs). For example, breast CTCs were found to express both epithelial and mesenchymal markers demonstrating EMT features (31). We also showed that CTCs are highly heterogeneous population in breast cancer and have similar phenotypes to STCs: various combinations of the stem and EMT features or the absence of these marks (32). The similarity of STC 
and CTC phenotypes may indicate the high ability of STCs to intravasation (33).

The relationship between stemness and EMT in tumor cells is widely discussed (34); however, opinions about causal relationships between these processes are contradictory. The recent study showed that EMT inhibition results in the acquisition of stemness and the initiation of breast cancer metastasis. In contrast, EMT activation suppressed stem features (27). These findings are in agreement with our results that eSTCs are associated with breast cancer metastasis. Moreover, it was found that fibroblast-like cells with EMT features partially maintain the polarity, attach tightly to the extracellular matrix, and remain quiescent. It was assumed that these cells may irreversibly transform to cancer-associated fibroblasts (35).

\section{CONCLUSIONS}

STCs demonstrate morphological diversity and phenotypical heterogeneity in stem and EMT features. STCs with epithelial morphology are associated with breast cancer metastasis and probably demonstrate a hybrid (metastable) EMT phenotype. Given these findings, eSTCs represent an attractive object in the study of mechanisms and key features that are typical of metastatic "seeds." In general, the determination of eSTCs in histological sections of breast tumors may be considered as an available prognostic marker of metastasis.

\section{REFERENCES}

1. Aceto N, Bardia A, Miyamoto DT, Donaldson MC, Wittner BS, Spencer JA, et al. Circulating tumor cell clusters are oligoclonal precursors of breast cancer metastasis. Cell. (2014) 158:1110-22. doi: 10.1016/j.cell.2014.07.013

2. Psaila B, Lyden D. The metastatic niche: adapting the foreign soil. Nat Rev Cancer. (2009) 9:285-93. doi: 10.1038/nrc2621

3. Au SH, Storey BD, Moore JC, Tang Q, Chen YL, Javaid S, et al. Clusters of circulating tumor cells traverse capillary-sized vessels. Proc Natl Acad Sci USA. (2016) 113:4947-52. doi: 10.1073/pnas.1524448113

4. Grigore $\mathrm{AD}$, Jolly $\mathrm{MK}$, Jia D, Farach-Carson MC, Levine H. Tumor budding: the name is EMT. partial EMT. J Clin Med. (2016) 5:51. doi: $10.3390 /$ jcm5050051

5. Lakhani SR, Ellis IO, Schnitt SJ, Tan PH, van de Vijver MJ. World Health Organization (WHO) Classification of Tumours of the Breast, 4 ed. Lyon: IARC Press (2012).

6. Zavyalova MV, Perelmuter VM, Vtorushin SV, Denisov EV, Litvyakov NV, Slonimskaya EM, et al. The presence of alveolar structures in invasive ductal NOS breast carcinoma is associated with lymph node metastasis. Diagn Cytopathol. (2013) 41:279-82. doi: 10.1002/dc.21852

7. Denisov EV, Skryabin NA, Gerashchenko TS, Tashireva LA, Wilhelm J, Buldakov MA, et al. Clinically relevant morphological structures in breast cancer represent transcriptionally distinct tumor cell populations with varied degrees of epithelial-mesenchymal transition and CD44(+)CD24(-) stemness. Oncotarget. (2017) 8:61163-80. doi: 10.18632/oncotarget. 18022

8. Gerashchenko TS, Novikov NM, Krakhmal NV, Zolotaryova SY, Zavyalova MV, Cherdyntseva NV, et al. Markers of cancer cell invasion: are they good enough? J Clin Med. (2019) 8:1092. doi: 10.3390/jcm8081092

9. Chikina AS, Alexandrova AY. The cellular mechanisms and regulation of metastasis formation. Mol Biol. (2014) 48:16580. doi: 10.1134/S0026893314020046

\section{DATA AVAILABILITY STATEMENT}

The datasets generated for this study are available on request to the corresponding author.

\section{ETHICS STATEMENT}

The studies involving human participants were reviewed and approved by Ethic Committee of the Cancer Research Institute, Tomsk NRMC (the approval number is 8 , on 17 June 2016). The patients/participants provided their written informed consent to participate in this study.

\section{AUTHOR CONTRIBUTIONS}

VP and MZ designed the research. MZ performed histology image analysis. LT, OS, and EK performed immunofluorescence analysis, score, and analysis of STCs in breast cancer samples. LT prepared confocal images. LT and TG performed data analysis. LT, ED, and VP drafted the work. VP supervised the study and the final approval of the version to be published.

\section{FUNDING}

This study was supported by the Russian Science Foundation (grant \#19-75-30016).

10. Krakhmal NV, Zavyalova MV, Denisov EV, Vtorushin SV, Perelmuter VM. Cancer invasion: patterns and mechanisms. Acta Nat. (2015) 7:1728. doi: 10.32607/20758251-2015-7-2-17-28

11. Zavyalova MV, Denisov EV, Tashireva LA, Gerashchenko TS, Litviakov NV, Skryabin NA, et al. Phenotypic drift as a cause for intratumoral morphological heterogeneity of invasive ductal breast carcinoma not otherwise specified. BioResearch Open Access. (2013) 2:148-54. doi: 10.1089/biores.2012.0278

12. Hammond ME, Hayes DF, Dowsett M, Allred DC, Hagerty KL, Badve S, et al. American Society of Clinical Oncology/College of American Pathologists guideline recommendations for immunohistochemical testing of estrogen and progesterone receptors in breast cancer (unabridged version). Arch Pathol Lab Med. (2010) 134:e48-72. doi: 10.1043/1543-2165-134.7.e48

13. Coates AS, Winer EP, Goldhirsch A, Gelber RD, Gnant M, PiccartGebhart $\mathrm{M}$, et al. Tailoring therapies-improving the management of early breast cancer: St Gallen International Expert Consensus on the Primary Therapy of Early Breast Cancer 2015. Ann Oncol. (2015) 26:153346. doi: 10.1093/annonc/mdv221

14. Peinado H, Olmeda D, Cano A. Snail, Zeb and bHLH factors in tumour progression: an alliance against the epithelial phenotype? Nat Rev Cancer. (2007) 7:415-28. doi: 10.1038/nrc2131

15. Huang RY, Wong MK, Tan TZ, Kuay KT, Ng AH, Chung VY, et al. An EMT spectrum defines an anoikis-resistant and spheroidogenic intermediate mesenchymal state that is sensitive to e-cadherin restoration by a src-kinase inhibitor, saracatinib (AZD0530). Cell Death Dis. (2013) 4:e915. doi: 10.1038/cddis.2013.442

16. Lindley LE, Briegel KJ. Molecular characterization of TGFbeta-induced epithelial-mesenchymal transition in normal finite lifespan human mammary epithelial cells. Biochem Biophys Res Commun. (2010) 399:659-64. doi: 10.1016/j.bbrc.2010.07.138

17. Hou Y, Guo H, Cao C, Li X, Hu B, Zhu P, et al. Single-cell triple omics sequencing reveals genetic, epigenetic, and transcriptomic heterogeneity in hepatocellular carcinomas. Cell Res. (2016) 26:304-19. doi: 10.1038/cr.2016.23 
18. Foley JW, Zhu C, Jolivet P, Zhu SX, Lu P, Meaney MJ, et al. Gene expression profiling of single cells from archival tissue with laser-capture microdissection and Smart-3SEQ. Genome Res. (2019) 29:1816-25. doi: 10.1101/gr.2348 07.118

19. Casasent AK, Schalck A, Gao R, Sei E, Long A, Pangburn W, et al. Multiclonal invasion in breast tumors identified by topographic single cell sequencing. Cell. (2018) 172:205-17 e12. doi: 10.1016/j.cell.2017.12.007

20. Heimann R, Hellman S. Aging, progression, and phenotype in breast cancer. J Clin Oncol. (1998) 16:2686-92. doi: 10.1200/JCO.1998.16.8.2686

21. Mayor R, Etienne-Manneville S. The front and rear of collective cell migration. Nat Rev Mol Cell Biol. (2016) 17:97-109. doi: 10.1038/nrm.2015.14

22. Slattum G, Gu Y, Sabbadini R, Rosenblatt J. Autophagy in oncogenic K-Ras promotes basal extrusion of epithelial cells by degrading S1P. Curr Biol. (2014) 24:19-28. doi: 10.1016/j.cub.2013.11.029

23. Gudipaty SA, Rosenblatt J. Epithelial cell extrusion: pathways and pathologies. Semin Cell Dev Biol. (2017) 67:132-40. doi: 10.1016/j.semcdb.2016.05.010

24. Lambert AW, Pattabiraman DR, Weinberg RA. Emerging biological principles of metastasis. Cell. (2017) 168:67091. doi: 10.1016/j.cell.2016.11.037

25. Nieto MA, Huang RY, Jackson RA, Thiery JP. Emt: 2016. Cell. (2016) 166:2145. doi: 10.1016/j.cell.2016.06.028

26. Jolly MK, Boareto M, Huang B, Jia D, Lu M, Ben-Jacob E, et al. Implications of the hybrid epithelial/mesenchymal phenotype in metastasis. Front Oncol. (2015) 5:155. doi: 10.3389/fonc.2015.00155

27. Nan X, Wang J, Liu HN, Wong STC, Zhao H. Epithelial-mesenchymal plasticity in organotropism metastasis and tumor immune escape. J Clin Med. (2019) 8:E747. doi: 10.3390/jcm8050747

28. Clark AG, Vignjevic DM. Modes of cancer cell invasion and the role of the microenvironment. Curr Opin Cell Biol. (2015) 36:1322. doi: 10.1016/j.ceb.2015.06.004

29. Bronsert P, Enderle-Ammour K, Bader M, Timme S, Kuehs M, Csanadi A, et al. Cancer cell invasion and EMT marker expression: a three-dimensional study of the human cancer-host interface. J Pathol. (2014) 234:41022. doi: 10.1002 /path.4416

30. Zhao Z, Zhu X, Cui K, Mancuso J, Federley R, Fischer K, et al. In vivo visualization and characterization of epithelialmesenchymal transition in breast tumors. Cancer Res. (2016) 76:2094-104. doi: 10.1158/0008-5472.CAN-15-2662

31. Yu M, Bardia A, Wittner BS, Stott SL, Smas ME, Ting DT, et al. Circulating breast tumor cells exhibit dynamic changes in epithelial and mesenchymal composition. Science. (2013) 339:580-4. doi: 10.1126/science.1228522

32. Kaigorodova EV, Savelieva OE, Tashireva LA, Tarabanovskaya NA, Simolina EI, Denisov EV, et al. Heterogeneity of circulating tumor cells in neoadjuvant chemotherapy of breast cancer. Molecules. (2018) 23:E727. doi: 10.3390/molecules23040727

33. Zavyalova MV, Denisov EV, Tashireva LA, Savelieva OE, Kaigorodova EV, Krakhmal NV, et al. Intravasation as a key step in cancer metastasis. Biochemistry. (2019) 84:762-72. doi: 10.1134/S0006297919070071

34. Brabletz T, Kalluri R, Nieto MA, Weinberg RA. EMT in cancer. Nat Rev Cancer. (2018) 18:128-34. doi: 10.1038/nrc.2017.118

35. Kalluri R, Zeisberg M. Fibroblasts in cancer. Nat Rev Cancer. (2006) 6:392401. doi: $10.1038 / \mathrm{nrc1877}$

Conflict of Interest: The authors declare that the research was conducted in the absence of any commercial or financial relationships that could be construed as a potential conflict of interest.

Copyright (c) 2020 Tashireva, Zavyalova, Savelieva, Gerashchenko, Kaigorodova, Denisov and Perelmuter. This is an open-access article distributed under the terms of the Creative Commons Attribution License (CC BY). The use, distribution or reproduction in other forums is permitted, provided the original author(s) and the copyright owner(s) are credited and that the original publication in this journal is cited, in accordance with accepted academic practice. No use, distribution or reproduction is permitted which does not comply with these terms. 